DOI: $10.17516 / 1999-494 X-0317$

УДК 621.565.83:629.7

\title{
Heat Pipe System as a Component of Spacecraft Electronics
}

\author{
Nikita Yu. Sokolova, \\ Vladimir A. Kulagin*b and Dmitry A. Nesterov ${ }^{\mathrm{c}}$ \\ anformation Satellite Systems - Reshetnev Company \\ Zheleznogorsk, Russian Federation \\ ${ }^{b}$ Siberian Federal University \\ Krasnoyarsk, Russian Federation \\ ${ }^{c}$ Institute of Computational Modeling SB RAS \\ Division of FRC KSC SB RAS \\ Krasnoyarsk, Russian Federation
}

Received 14.04.2021, received in revised form 11.05.2021, accepted 21.06.2021

\begin{abstract}
We report on the results of optimizing a single flat heat pipe into an arrangement of heat pipes. A comparison is drawn at the same temperatures and occupied volumes and for a specific maximum temperature of radio-electronic devices. The end result of our studies is that the limiting heat transfer capacity has been found for a single heat pipe and two- and three-level heat pipe assemblies with various heat transfer media. Versatility of the mathematical model enhanced by the optimization method has been proved.
\end{abstract}

Keywords: flat heat pipe, heat pipe system, cooling of electronics, high thermal power density.

Citation: Sokolov N.Yu., Kulagin V.A., Nesterov D.A. Heat pipe system as a component of spacecraft electronics, J. Sib. Fed. Univ. Eng. \& Technol., 2021, 14(4), 363-377. DOI: 10.17516/1999-494X-0317

(C) Siberian Federal University. All rights reserved

This work is licensed under a Creative Commons Attribution-Non Commercial 4.0 International License (CC BY-NC 4.0).

* Corresponding author E-mail address: vak-sfu@mail.ru 


\title{
Система тепловых труб в составе \\ радиоэлектронного оборудования \\ космического аппарата
}

\author{
Н.Ю. Соколов ${ }^{\mathrm{a}}$, В.А. Кулагин ${ }^{0}$, Д.А. Нестеров \\ ${ }^{a}$ Информационные спутниковые системь \\ им. акад. М.Ф. Решетнева \\ Российская Федераџия, Железногорск \\ ${ }^{6}$ Сибирский федеральный университет \\ Российская Федерачия, Красноярск \\ ${ }^{8}$ Институт вычислительного моделирования СО РАН - \\ обособленное подразделение ФИЦ КНЦ СО РАН \\ Российская Федерачия, Красноярск
}

\begin{abstract}
Аннотация. Изложены результаты оптимизации одиночной плоской тепловой трубы в систему тепловых трубок. Сравнение проводится при одинаковых температурах и занимаемых объемах и для конкретной максимальной температуры радиоэлектронных устройств. Конечным результатом исследований является то, что предельная способность теплопередачи была найдена для одиночной тепловой трубы и двух- и трехуровневых тепловых труб в сборе с различными теплоносителями. Доказана универсальность математической модели, усиленной методом оптимизации.
\end{abstract}

Ключевые слова: плоская тепловая труба, система тепловых труб, охлаждение радиоэлектронной аппаратуры, высокая плотность тепловой мощности.

Цитирование: Соколов, Н.Ю. Система тепловых труб в составе радиоэлектронного оборудования космического аппарата / Н.Ю. Соколов, В.А. Кулагин, Д.А. Нестеров // Журн. Сиб. федер. ун-та. Техника и технологии, 2021, 14(4). C. $363-377$. DOI: $10.17516 / 1999-494 X-0317$

\section{Введение}

С каждым годом информационный поток становится все больше, поэтому именно спутниковые системы связи представляют собой одну из наиболее динамично развивающихся разновидностей космических информационных систем. Одним из непременных условий надежного функционирования космического аппарата (КА) и его служебных систем, а также оборудования полезной нагрузки является обеспечение необходимого теплового режима всех его компонентов.

В статье Амира Фагри [1] представлен подробный обзор тепловых трубок, включая историческую перспективу, принципы работы, типы тепловых труб, характеристики тепловых труб, ограничения тепловых труб, запуск и отключение тепловых труб с замораживанием, анализ и моделирование тепловых трубок, а также различные тепловые характеристики их приложений. В статье [2] дан обзор тепловых трубок, особенно используемых в различных космических полетах. Обсуждаются исторические перспективы, принципы работы, типы тепловых труб. В работе [3] представлено практическое исследование современной технологии тепловых трубок, в котором обсуждается, как ее можно оптимизировать для использования в более широком масштабе. Эта книга предлагает обзор теории тепломассопереноса, относящейся к производительности тепловых труб, особенно в приложениях с высоким тепловым пото- 
ком и в ситуациях, в которых возможна любая комбинация неравномерной тепловой нагрузки, ограниченного воздушного потока над тепловыделяющими компонентами, а также ограничений по площади или весу. Решаются ключевые проблемы внедрения, включая балансировку нагрузки, характеристики материалов, диапазоны рабочих температур, тепловое сопротивление и рабочую ориентацию. Представлены математические модели для расчета ограничений теплопередачи и температурного градиента как для высокотемпературных, так и для низкотемпературных тепловых труб. В книге результаты расчетов сравниваются с имеющимися экспериментальными данными. Она также включает в себя серию компьютерных программ, разработанных автором для поддержки представленных данных, помощи в проектировании и прогнозирования производительности.

Перспективы развития информационных спутниковых систем в России связаны с созданием негерметичных космических аппаратов. Такие аппараты уже вошли в состав глобальной навигационной системы ГЛОНАСС и в недалеком будущем станут ее основой $[4,5]$. Одновременно идет разработка новых моделей КА с увеличенной мощностью более 5 кВТ, сроком активного существования более 12 лет и с повышенными точностными навигационно-временными характеристиками. Одной из главных проблем является обеспечение оптимального теплового режима радиоэлектронной аппаратуры (РЭА) на борту КА. В условиях постоянно возрастающей мощности бортовой аппаратуры и необходимости уменьшения ее объема и массы обеспечение работы КА возможно только за счет увеличения эффективности работы систем отвода тепла. Основной принцип функционирования системы обеспечения теплового режима заключается в поддержании требуемого диапазона температур на посадочных местах тепловыделяющего оборудования космического аппарата КА при заданных энергопотреблениях. От этого зависит долговечность и надежность функционирования спутника в целом.

Обеспечение теплового режима современных КА негерметичного исполнения, например спутников связи с увеличенным сроком активного существования, достигается с помощью тепловых труб. Для минимизации массоэнергетических параметров системы целесообразно использовать принцип естественной, нерегулируемой передачи тепловой мощности с более высокого температурного уровня на низкий. Это обеспечивается отбором тепловой мощности, выделяемой аппаратурой, от ее посадочных мест на термоплатах (панелях), передачей этой мощности на радиационный теплообменник (РТО) и отводом ее с РТО в окружающее пространство. Наиболее предпочтительным средством реализации этого принципа являются тепловые трубы (ТТ), работающие на основе замкнутого испарительно-конденсационного цикла. ТТ отличаются высокой эффективной теплопроводностью, в десятки раз превышающей теплопроводность металлов [6, 7]. Они обеспечивают эффективный отвод тепла от теплонагруженных электрорадиоизделий (ЭРИ), позволяют трансформировать плотность тепловых потоков, разнести в пространстве источник и приемник теплоты, стабилизировать температуру и уменьшить неравномерность температурного поля по конструкции радиоэлектронной аппаратуры. В отличие от систем, имеющих жидкостный контур охлаждения с принудительной циркуляцией теплоносителя, системы из тепловых труб не потребляют электроэнергии, значительно выигрывают в массе и надежности. Поэтому одним из перспективных научно-технических направлений в развитии конструкции современной РЭА КА признано внедрение тепловых труб. В настоящее время разработан новый класс плоских ТТ, встраиваемых в несущую конструк- 
цию блока РЭА, - гипертеплопроводящая секция (ГТПС), отличающаяся от прочих ТТ оригинальной конструкцией корпуса. По сути все современные научные работы в данной области направлены на переработку материала 1960-70-х годов, увеличение отводимой мощности от ЭРИ обеспечивают за счет параллельного соединения ТТ либо за счет увеличения объема пористой структуры ТТ. Зависимость максимально отводимой мощности от длины ТТ является нелинейной, поэтому при определенных параметрах последовательные и последовательно-параллельные схемы соединения ТТ превосходят схемы прямого одиночного или параллельного соединения ТТ. Это позволяет оптимально решать многие инженерные задачи, связанные с передачей тепла с минимальными потерями, охлаждением и термостатированием или термостабилизацией различных объектов, использующихся как на Земле, так и в космическом пространстве. Важно также развитие методов экспериментального исследования гидродинамики двухфазного газожидкостного течения в микроканале, получение закономерностей режимов течения и определение границ режимов течения, определение потерь на трение для каналов с различной ориентацией относительно вектора силы тяжести [8]. Современные искусственные спутники Земли для связи и телерадиовещания представляют собой КА, оснащенные крупногабаритным негерметичным приборным отсеком в виде блочно-модульной конструкции на основе плоских прямоугольных сотовых панелей [9], несущих на себе теплонагруженные приборы радиоэлектронной бортовой аппаратуры (БА) [10].

Отсутствие в конструкциях ТТ движущихся механических частей, использование для движения теплоносителя капиллярных сил, высокая плотность передаваемого теплового потока при минимальных перепадах температур (термических сопротивлениях) и автономность работы каждой из них, возможность резервирования, минимальное обслуживание и малый удельный вес, бесшумная работа создают им преимущества и обеспечивают надежность и долговечность перед иными способами термостатирования заданного объекта на борту КА. Панели с вмонтированными в них ТТ - надежная защита от ударов микрометеоритов [5]. Изотермализирующая природа ТТ уменьшает температуры мощных компонентов тепловыделяющего оборудования, увеличивая таким образом надежность их работы. Выигрыш в массе дает возможность распределения массы КА в пользу систем энергопитания, двигателей и полезной нагрузки $[11,12]$. Существуют различные методы проверки работоспособности ТТ - термовакуумные испытания (ТВИ) каждого образца, экспресс-контроль в составе РЭА и т.п. В этой связи важно совершенствование испытательного оборудования, обеспечивающего высокую точность определения характеристик ТТ, что в итоге обеспечит надежность и долговечность работы КА на орбите [13-15].

Основная идея данной работы заключается в разработке новой методики уменьшения массогабаритных характеристик приборов КА, основанного на применении новой математической модели ТТ для описания термодинамических характеристик систем, состоящих из ТТ. Главное отличие новой математической модели от существующих, например изложенной в [16-18], в том, что критерием увеличения ТТ в системе ТТ является уменьшение массы системы ТТ при добавлении ТТ и уменьшение температурного запаса ЭРИ. Если конструктив рамки отработан и унифицирован, то большой температурный запас $\left(>5^{\circ} \mathrm{C}\right.$ с учетом срока активного существования (САС КА) для ЭРИ во время наземной экспериментальной отработки БА может означать только одно - избыточную массу рамки. Для обеспечения тепловых режимов ЭРИ в блоках с 


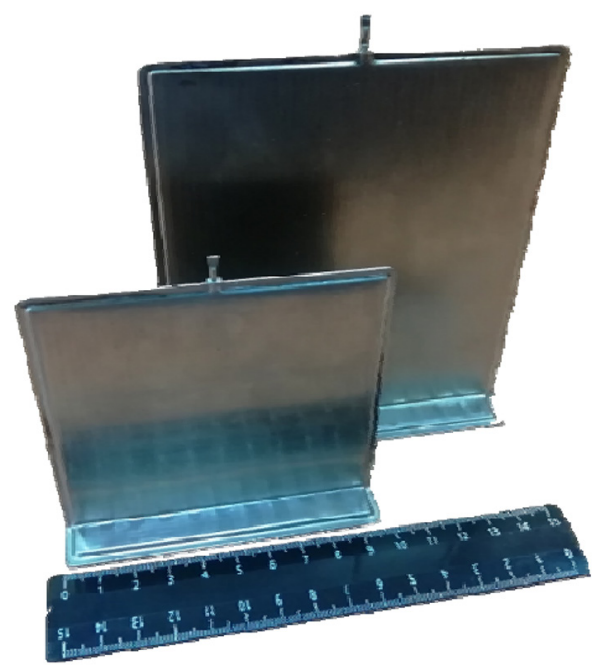

Рис. 1. Внешний вид ГТПС

Fig. 1. External appearance of HHCE

тепловыделением 16-160 Вт в АО «ИСС» разработан конструктив рамок со встроенными гипертеплопроводящими секциями (ГТПС) [19].

Результаты расчета математической модели подтверждены экспериментально на вновь созданной системе ТТ (состоящей из двух ГТПС). Разработанный в итоге блок прибора КА обладает превосходными массогабаритными характеристиками. Система термостабилизации (СТР) современных КА, выпускаемых АО «ИСС», обеспечивает температуры посадочных мест БА в диапазоне от минус 10 до плюс $40^{\circ} \mathrm{C}$. Максимальная рабочая температур ЭРИ определена техническими условиями (ТУ) ЭРИ производителем, также производителем определена максимальная рабочая температура ЭРИ с учетом срока активного существования (САС) КА. Конструкция БА должна обеспечивать тепловые режимы всех ЭРИ с учетом САС. Масса ТТ увеличивается непропорционально длине ТТ, что можно заметить на рис. 1, построенном по математической модели Чи [18, 20].

\section{Существующие математические модели TT,}

\section{нелинейная зависимость отводимой тепловой мощности от длины ТТ}

Стационарное ламинарное течение несжимаемой жидкости с постоянной вязкостью $\mu$ в круглой трубе радиуса а описывается уравнением Хагена-Пуазейля [4]. Это уравнение связывает скорость жидкости $v_{r}$ на радиусе $r$ с перепадом давления $P_{2}-P_{1}$ на участке трубы длиной $\ell$ :

$$
v_{r}=\frac{\mathrm{a}}{4 \mu}\left[1-\left(\frac{r}{\mathrm{a}}\right)^{2}\right] \frac{P_{2}-P_{1}}{\ell}
$$

Скорость по радиусу изменяется по закону параболы от максимального значения на оси трубы до нулевого на стенке. Средняя по сечению скорость равна:

$$
v=\frac{\mathrm{a}^{2}}{8 \mu} \frac{\left(P_{2}-P_{1}\right)}{\ell} .
$$


Массовый расход $\dot{m}$, протекающий через трубу за секунду, равен:

$$
\dot{m}=\rho S=\rho v \pi a^{2}=\frac{\rho \pi a^{4}}{8 \mu} \frac{\left(P_{2}-P_{1}\right)}{\ell},
$$

где $S$ - объемный расход.

Режим течения в жидкой фазе в тепловой трубе почти всегда ламинарный. Поскольку каналы для прохода жидкости в общем случае не будут прямолинейны, не будут иметь круглое поперечное сечение и, кроме того, будут соединяться между собой, то уравнение Хагена-Пуазейля должно быть модифицировано с учетом этих обстоятельств.

$$
\dot{m}=\frac{\pi\left(r_{\mathrm{W}}^{2}-r_{\mathrm{V}}^{2}\right) \varepsilon r_{\mathrm{C}}^{2} \rho_{\mathrm{l}}}{8 \mu_{1}} \frac{\Delta P_{1}}{\ell_{\mathrm{eff}}},
$$

где $\varepsilon$ - пористость фитиля; $r_{\mathrm{w}}$ - радиус до стенки ТТ; $r_{\mathrm{v}}-$ радиус газового канала ТT; $r_{\mathrm{c}}$ эффективный радиус поры; $\ell_{\text {eff }}$ - полная эффективная длина канала для прохода жидкости. Связывая $\dot{m}$ с тепловым потоком соотношением $Q=\dot{m} L$, где $L-$ скрытая теплота испарения, получаем:

$$
\Delta P_{1}=\frac{8 \mu_{1} Q \ell_{\mathrm{eff}}}{\pi\left(r_{w}^{2}-r_{v}^{2}\right) \varepsilon r_{c}^{2} \rho_{1} L} .
$$

Для пористой среды уравнение (5) обычно записывают в виде

$$
\Delta P_{1}=\frac{b \mu_{1} Q \ell_{\mathrm{eff}}}{\pi\left(r_{w}^{2}-r_{v}^{2}\right) \varepsilon r_{c}^{2} \rho_{1} L}
$$

где $b$ - безразмерная постоянная, учитывающая поправку на извилистость каналов, равная примерно 10-20. Это уравнение содержит три постоянные $-b, r$ и $\varepsilon$, каждую из которых трудно измерить. Поэтому обычно при расчетах $\Delta P_{1}$ в подобного типа фитилях используют формулу Дарси, которая записывается как

$$
\Delta P_{1}=\frac{\mu_{1} \ell_{\text {eff }} \dot{m}}{\rho_{1} K A},
$$

где $K$ - проницаемость фитиля; $A$ - поперечное сечение.

Формула Дарси представляет собой уравнение Хагена-Пуазейля, содержащее поправочные члены, объединенные параметром $K$, учитывающим размер пор, распределение и извилистость каналов. Проницаемость $K$ легко поддается измерению.

Капиллярное ограничение (ограничение по смачиваемости фитиля) [4]. Для того чтобы ТТ работала, необходимо, чтобы удовлетворялось соотношение:

$$
\begin{aligned}
& \Delta P_{c} \geq \Delta P_{1}+\Delta P_{v}+\Delta P_{g}, \\
& \Delta P_{g}=\rho_{\mathrm{l}} g \ell_{\mathrm{eff}} \sin \varphi, \\
& \Delta P_{\mathrm{c}}=\frac{2 \sigma \cos \theta}{r_{c}}, \\
& \Delta P_{v}=\frac{8 \mu_{v} \ell_{\mathrm{eff}} \dot{m}}{\pi r_{v}^{4}}, \\
& \Delta P_{1}=\frac{\mu_{1} \ell_{\mathrm{eff}} \dot{Q}}{\rho_{1} L A K},
\end{aligned}
$$

где $\varphi$ - угол наклона ТТ; $g$ - ускорение свободного падения; $\theta$ - угол смачивания; $\sigma$ - поверхностная энергия; $\mu_{v}$ - динамическая вязкость пара; $\dot{Q}$ - максимально отводимая тепловая мощ- 
ность. Формулу (8.1) можно переписать в более простом виде, если принять следующие допущения:

1) ТТ находится в горизонтальном положении или в условиях невесомости;

2) перепадом давления в паровом потоке можно пренебречь;

3) свойства жидкости не меняются вдоль трубы;

4) фитиль является однородным.

$$
\Delta P_{c}=\Delta P_{1}
$$

или

$$
\frac{2 \sigma \cos \theta}{r_{c}}=\frac{\mu_{1} \ell_{\text {eff }} \dot{Q}}{\rho_{1} L A K}
$$

Перепишем уравнение (9.1) таким образом, чтобы выделить характер зависимости отводимой тепловой мощности от длины ТТ.

$$
\dot{Q}=\frac{\mathrm{C}}{\ell_{\mathrm{eff}}}
$$

где

$$
\mathrm{C}=\frac{2 \sigma \cos \theta \rho_{\mathrm{l}} L A K}{\mu_{\mathrm{l}} r_{C}} .
$$

Соотношение (10) показывает обратную пропорциональность отводимой тепловой мощности от длины ТТ, т.е. зависимость нелинейная. Очевидно, что уравнение (10) представляет собой уравнение равнобочной гиперболы. В работе [5] предложена математическая модель оптимизации последовательного соединения одномерных (цилиндрических) ТТ, данную модель также можно применить к плоским ТТ в случае равномерного подвода и отвода тепловой мощности.

Поскольку в формуле (8.1) все величины справа суммируются, а параметр $\ell_{\text {eff }}$ находится в числителе, отмена допущений, принятых для вывода соотношения (10), увеличит нелинейную зависимость отводимой тепловой мощности от длины ТТ. Также из формул (8.1)-(8.5) следует, что скорость продвижения фронта жидкости в процессе заполнения фитиля будет уменьшаться по мере уменьшением разницы давления между максимальным капиллярным давлением и текущим давлением в капилляре. Эффективная теплопроводность капиллярных ТТ в 10-100 раз превышает теплопроводность металлов, и потенциально ТТ могут стать основой высокоэффективного теплопередающего тракта в задачах современного приборостроения, а также для передачи тепловой энергии в глобальных масштабах (ТЭЦ и пр.).

\section{Существующая математическая модель ГТПС, программный комплекс для расчета предельных характеристик}

Прорывным научно-техническим решением, которое обеспечило эффективный отвод тепла от всех ЭРИ бортовой РЭА, является ГТПС [19]. Новый класс тепловых труб представляет собой плоскую тонкую герметичную конструкцию, внутри которой находится фитиль из спеченного металлического порошка, заполненный жидким теплоносителем (рис. 1).

В фитиле также располагаются каналы для движения пара. Фитиль и паровые каналы обеспечивают движение теплоносителя в любом направлении в плоскости тепловой трубы. На

$$
-369-
$$


поверхности тепловой трубы произвольным образом могут располагаться источники и стоки тепла. В местах подвода тепла жидкость испаряется и движется по паровым каналам к области охлаждения, где пар конденсируется и движется обратно по фитилю. Скрытая теплота парообразования теплоносителя обеспечивает высокую эффективность передачи тепла. Движение рабочего вещества осуществляется за счет капиллярных сил.

Для обеспечения срока активного существования (САC) 140000 часов (15 лет) необходимо, чтобы применяемые в аппаратуре ЭРИ функционировали при температуре не более $55{ }^{\circ} \mathrm{C}$ при том, что на установочном месте бортовой РЭА гарантируется температура не более $40{ }^{\circ} \mathrm{C}$. На рис. 2 представлена конструктивная реализация ГТПС в бортовой РЭА КА.

В ИВМ СО РАН по заказу АО «ИСС» был разработан программный комплекс моделирования бортовой РЭА, который обеспечивает полный тепловой расчет бортовой РЭА, содержащей в своем составе блоки со встроенными ГТПС. Входными данными являются геометрические и теплофизические характеристики узлов прибора, расположение и мощность тепловыделяющих элементов, параметры тепловых контактов, характеристики для учета теплообмена за счет излучения и теплообмена с окружающей средой. При использовании ГТПС учитываются характеристики теплоносителя и материалов фитиля и корпуса тепловой трубы, параметры и геометрия пористой структуры. Предусмотрены различные варианты внешних условий и теплоотвода, соответствующие условиям испытаний РЭА при отработке или функционировании в составе КА на орбите: испытания отдельного блока или прибора в сборе, наличие атмосферы или вакуум, наличие гравитации. В качестве теплоотвода учитываются различные варианты: заданная температура основания, сотовая панель с охлаждающими магистралями, пластина с жидкостными каналами.

Выходными данными программного комплекса служат температуры элементов, тепловые поля конструкций блоков и стенок прибора, распределение температур поверхности теплоотводящего основания, изменение температуры жидкости по длине охлаждающих магистралей.
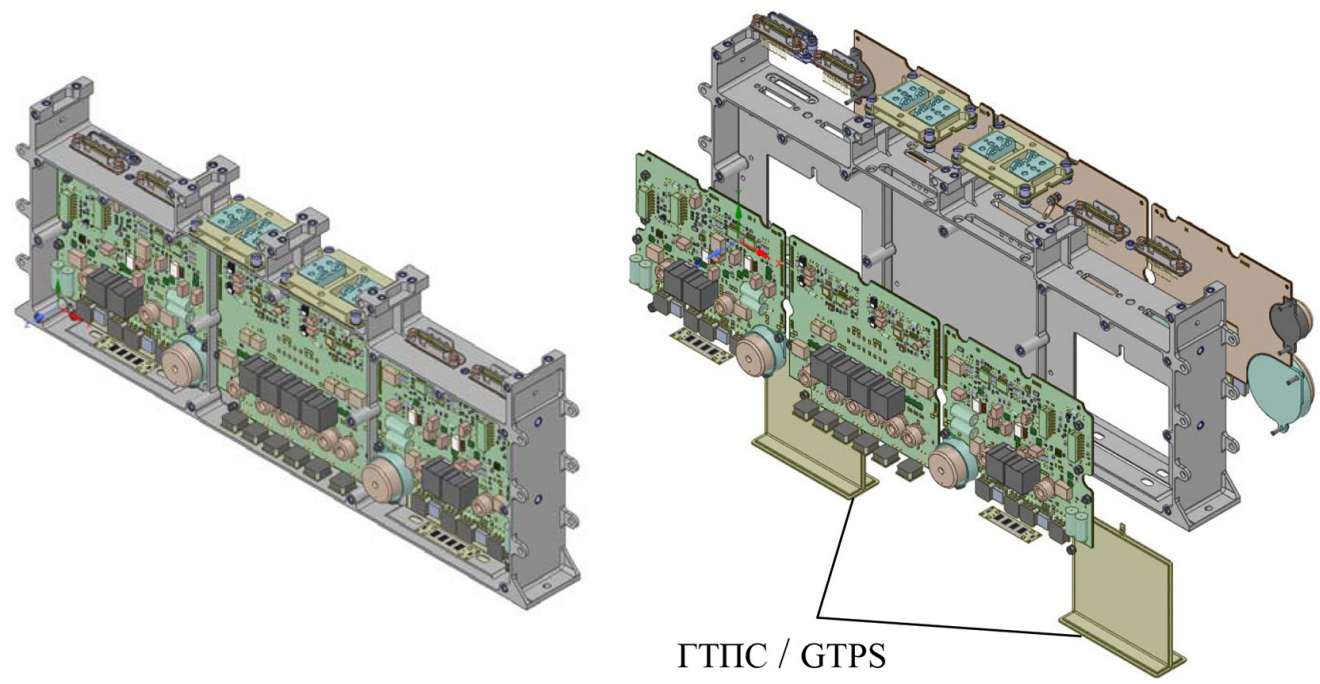

Рис. 2. Модель блока бортовой РЭА с интегрированными ГТПС

Fig. 2. Model of the on-board REA unit with integrated GTPS 
При наличии ГТПС определяются распределения давлений и скоростей паровой и жидкостной фаз теплоносителя, наличие зон осушения или замерзания теплоносителя.

Расчетный модуль программного комплекса использует в работе набор различных математических моделей для решения тепловой задачи. Моделирование теплопередачи в конструкциях блока и прибора без ГТПС сводится к совместному итерационному решению стационарных уравнений теплопроводности и уравнений лучистого теплообмена (при учете излучения). Для плоских тепловых труб были разработаны более сложные модели и алгоритмы, основанные на совместном решении двухмерных уравнений теплопроводности, уравнений сохранения массы жидкости и пара, дополненных уравнениями Дарси. В модели используются усредненные характеристики, которые отражают проницаемость внутренней структуры плоской тепловой трубы для пара и жидкости.

При функционировании ГТПС обычно конвективный механизм переноса тепла является доминирующим. Однако требуется учитывать случаи, когда конвективный перенос тепла становится неэффективным (при температурах теплоносителя, близких к точке замерзания) или в случае прекращения движения теплоносителя (осушение фитиля, замерзание теплоносителя или блокирование паровых каналов неконденсирующимися газами). В этом случае необходимо учитывать перенос тепла за счет теплопроводности конструкции ГТПС (корпуса и фитиля).

Система уравнений в области ГТПС имеет вид:

$$
\begin{aligned}
& \operatorname{div}(-\mathrm{d} \lambda \nabla \mathrm{T}(x, y))+g(x, y) T(x, y)+g_{c}(x, y)=f(x, y), \\
& \operatorname{div}\left(d_{h p} \frac{\rho_{v}}{\mu_{v}} K_{v} \nabla P_{v}\right)=-\frac{q(x, y)}{L}, \\
& \operatorname{div}\left(d_{h p} \frac{\rho_{1}}{\mu_{l}} K_{1} \nabla P_{1}\right)=\frac{q(x, y)}{L},
\end{aligned}
$$

где $T(x, y)$ - распределение температуры; $\mathrm{d} \lambda$ - произведение толщины и эффективной теплопроводности конструкции тепловой трубы (при отсутствии циркуляции теплоносителя); $g(x, y)$ и $f(x, y)$ - коэффициенты, учитывающие внешние потоки тепла (тепловыделение ЭРИ, сток тепла, слагаемые для радиационных потоков тепла и пр.); $q(x, y)$ - поглощаемое испаряющимся теплоносителем в тепловой трубе тепло (Вт/м²); $P_{1}(x, y)$ и $P_{v}(x, y)$ - давления в жидкостной и паровой фазах теплоносителя; $L$ - скрытая теплота парообразования теплоносителя; $\mu$ и $\rho$ - вязкость и плотность фаз теплоносителя; $K_{1}, K_{v}$ - коэффициенты (в общем случае тензоры), определяющие проницаемость капиллярной структуры тепловой трубы для жидкости и пара.

Для решения системы (11.1)-(11.3) дополнительно используется соотношение $\nabla \mathrm{P}_{\mathrm{v}}=\frac{\partial \mathrm{P}_{\mathrm{v}}}{\partial T_{\mathrm{v}}} \nabla \mathrm{T}_{\mathrm{v}}$, указывающее, что пар внутри тепловой трубы находится на линии насыщения. При этом зависимость производной давления насыщенного пара по температуре может задаваться как отдельная характеристика теплоносителя. Величина q(x, у) в общем случае сложным образом зависит от состояния теплоносителя и в том числе от распределения температуры $T(x, y)$. То есть уравнение (6) нелинейное, поэтому его решение является сложной задачей. Система уравнений (11.1)-(11.3) решается итерационно, при этом на каждой итерации после расчета распределения давлений жидкости и пара проверяется следующее условие:

$$
\begin{gathered}
P_{v}(x, y)-P_{1}(x, y)<P_{c}, P_{c}=\frac{2 \sigma \cos \theta}{r_{c}}, \\
-371-
\end{gathered}
$$


где $r_{c}$ - радиус пор фитиля; $\sigma$ - коэффициент поверхностного натяжения; $\theta$ - предельный угол смачивания материала фитиля теплоносителем. В области, где условие (12) не выполняется, фитиль осушается и поглощение тепла при испарении прекращается. Величина q в этой области принимается равной нулю для последующих итераций. Аналогично учитывается условие на замерзание теплоносителя: $T(x, y)>T_{0}$, где $T_{0}$ - температура замерзания.

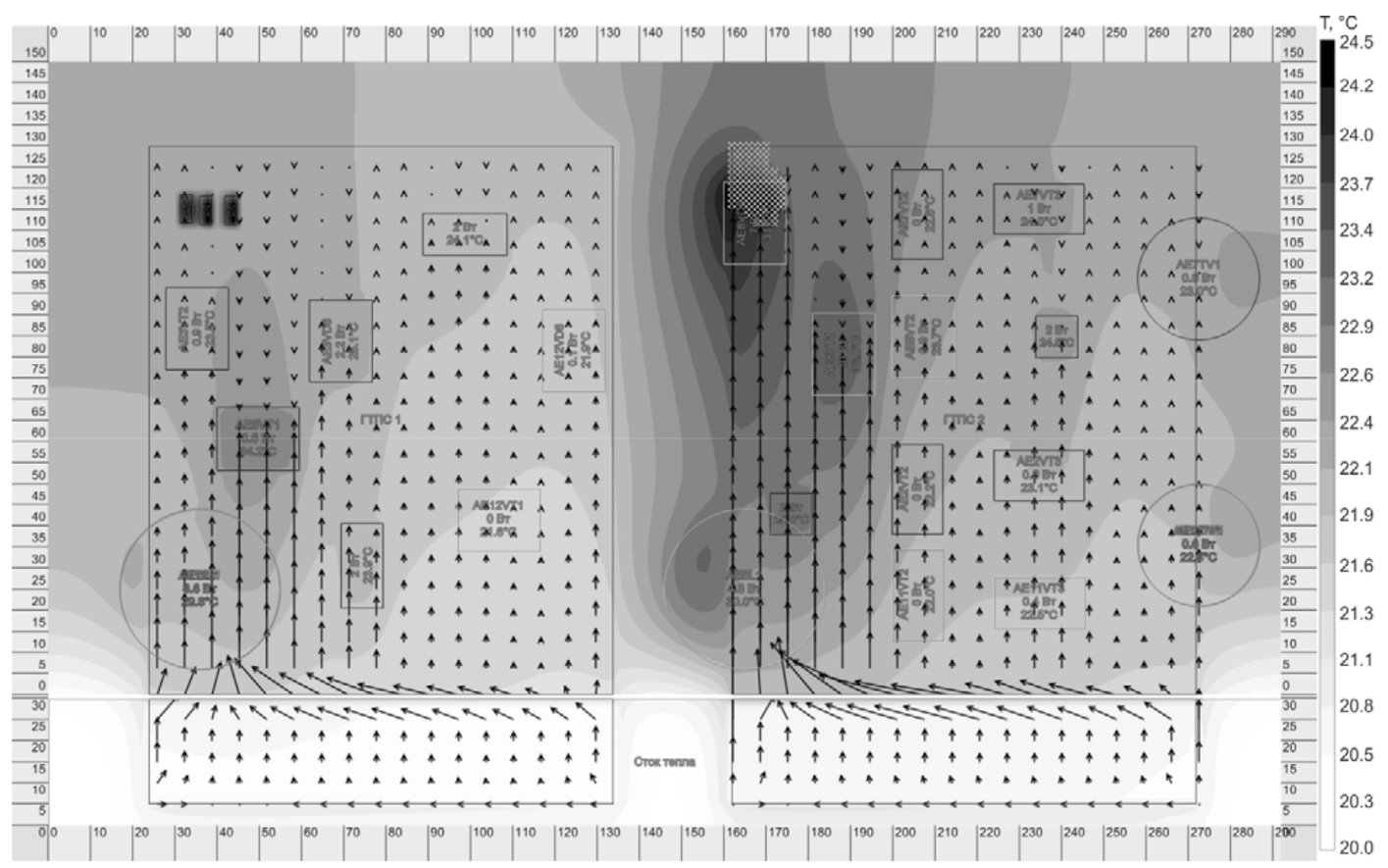

Рис. 3. Температура поверхности блока РЭА и поле скоростей жидкости в фитиле ГТПС

Fig. 3. Surface temperature of the CEA unit and the field of fluid velocities in the GTPS wick

Разработанный программный комплекс оснащен графическим интерфейсом, обеспечивающим удобную работу с программой, задание входных параметров, получение и анализ результатов расчетов. С целью увеличения точности расчета по результатам экспериментальной проверки ГТПС уточняются значения параметров математической модели (пористость, проницаемость и пр.). На рис. 3 показан пример расчета теплового поля поверхности блока РЭА со встроенными плоскими тепловыми трубами. Дополнительно показано поле скоростей жидкости в капиллярных структурах. На правой тепловой трубе (в левом верхнем углу) выделена область осушения фитиля, которое приводит к локальному увеличению температуры.

\section{Предельные характеристики плоских ТT}

\section{и их последовательное соединение}

В работе [4] проведен ряд экспериментов по определению максимального значения мощности и плотности мощности, которая может быть отведена с помощью ГТПС, при сконцентрированном подводе тепла, при различном расположении области подвода тепла. В качестве ис- 
точника тепла использовался транзистор, способный выделять тепловую мощность до 100 Вт

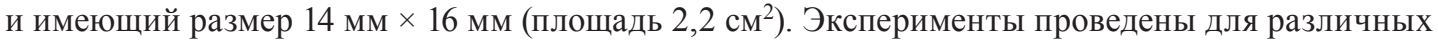
вариантов размещения источника тепла на поверхности тепловой трубы. При центральном расположении нагревателя расстояние до холодильника составляло 60 мм, при расположении в углу - 105 мм. Эксперименты проведены для горизонтальной ориентации и при температуре зоны конденсации минус $27^{\circ} \mathrm{C}$.

При расположенном в центре нагревателе осушение фитиля появилось при мощности нагревателя 24 Вт. Температура поверхности тепловой трубы составляла $35{ }^{\circ} \mathrm{C}$. Дальнейшее увеличение мощности нагревателя вызывает линейный рост температуры $1{ }^{\circ} \mathrm{C} / \mathrm{B}$. Для расположенного в углу нагревателя осушение фитиля обнаружено при 13 Вт при температуре поверхности тепловой трубы $35^{\circ} \mathrm{C}$. Дальнейшее увеличение мощности нагревателя вызывает линейный рост температуры $1,2^{\circ} \mathrm{C} / \mathrm{B}$.

Для вертикально ориентированной ГТПС отводимая от нагревателя мощность до осушения будет значительно меньше, что следует из уравнения (8.1). Также известно, что с уменьшением температуры возрастает динамическая вязкость жидкостей, а для воды в рабочем диапазоне температур РЭА КА существует точка фазового перехода, из чего следует значительное снижение отводимой от нагревателя мощности с уменьшением температуры.

Последовательное соединение плоских TT теоретически должно перераспределить тепловые потоки от ЭРИ. В случае частичного осушения границы соединения ТТ тепловой поток будет проводить металлический корпус, верхняя ТТ будет работать при более высокой температуре, что увеличит отводимую от нагревателя локальную мощность. Системное взаимное влияние ТТ на разных уровнях при последовательной и последовательно-параллельной схеме соединения позволяет определить новый термин - cистема тепловых труб (СТТ). В программном комплексе была смоделирована одиночная плоская ТТ (медная) с пористой структурой в виде столбиков и СТТ, состоящая из двух и трех уровней, соединенных встык (без увеличения толщины). Схемы расположения ТТ, зон отвода тепла и нагреватель показаны на рис. 4.

\section{Условия моделирования}

В области $100 \times 100$ мм на максимальном расстоянии от зоны охлаждения располагается

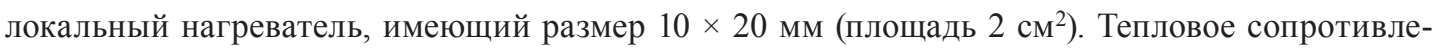
ние под нагревателем принято равным 0,28 Вт/K, что соответствует толщине клея 0,1 мм с теплопроводностью $1,8 \mathrm{BT} / \mathrm{M} \cdot \mathrm{K}$. Граница соединения ТТ имеет толщину $1 \cdot 10^{-3}$ м и теплопроводность $120 \mathrm{BT} / \mathrm{M} \cdot \mathrm{K}$. Поскольку в реальной топологии блока РЭА вблизи теплонагруженных ЭРИ (транзисторы, диоды, шунтирующие резисторы и пр.) располагаются прочие ЭРИ (резисторы, конденсаторы), необходимо учитывать, чтобы все ЭРИ имели запас по температуре. Пассивные ЭРИ расположены на расстоянии $\approx 5 \cdot 10^{-3}$ м от теплонагруженных ЭРИ. В случае осушения ТТ вблизи теплонагруженного ЭРИ появляется область высокой температуры.

Тепловыделяющие ЭРИ имеют максимальную рабочую температуру порядка $80{ }^{\circ} \mathrm{C}$ для $\mathrm{CAC} 140000$ ч, а пассивные ЭРИ имеют рабочую температуру порядка $55^{\circ} \mathrm{C}$ для САC 140000 ч. Поэтому сравнивать необходимо при одновременном выполнении двух условий: тепловыделяющее ЭРИ и пассивные ЭРИ должны находиться в рабочем диапазоне температур. Эксперименты проведены для вертикальной ориентации в поле действия гравитационных сил. Схема,

$$
-373-
$$




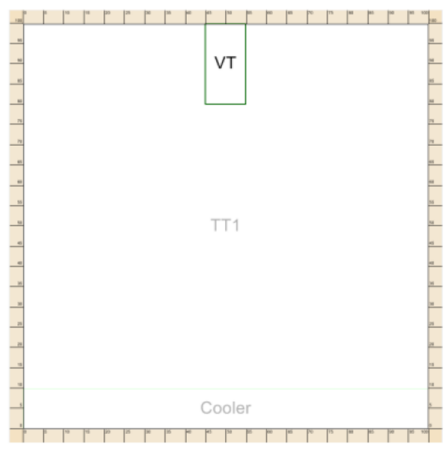

a)

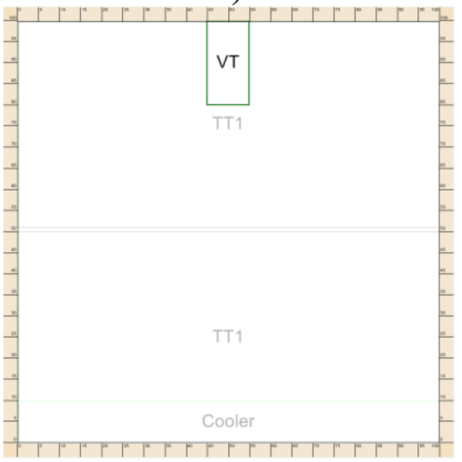

в)

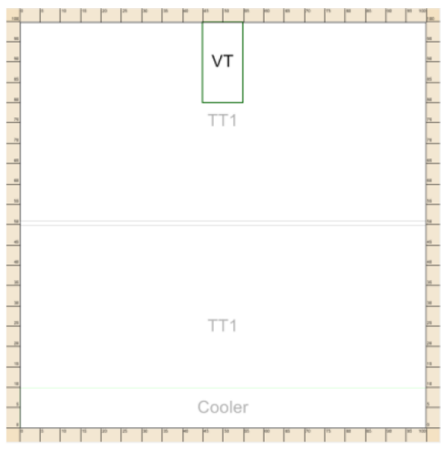

б)

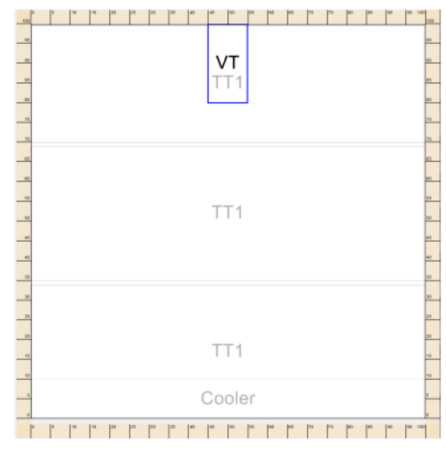

2)

Рис. 4. Схемы расположения ТТ: $a$ - одиночная плоская ТТ; $\sigma$ - СТТ 2-го уровня, оптимизированная под воду; в - СТТ 2-го уровня, оптимизированная под ацетон; 2 - СТТ 3-го уровня

Fig. 4. Layouts of TT: $a$-single flat TT; $\sigma$-CTT of the 2nd level, optimized for water; $b$ - CTT level 2, optimized for acetone; 2 - CTT level 3

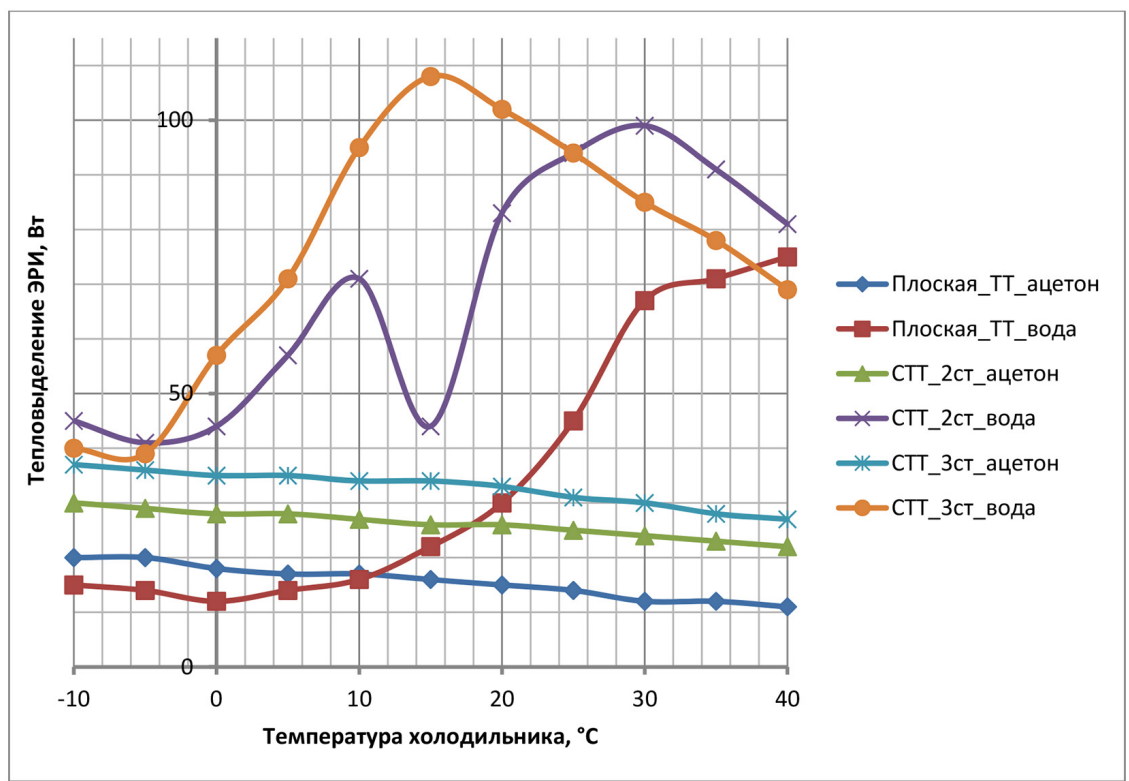

Рис. 5. Зависимость отводимой тепловой мощности нагревателя от температуры зоны охлаждения для разных схем соединения ТТ

Fig. 5. Thermal flux from the heater versus cooling zone temperature for various options of HP connection 
представленная на рис. 4б, была оптимизирована для воды методом перебора во всем диапазоне температур (рис. 5).

\section{Заключение}

Из графика для одиночной плоской ТТ с водой в качестве теплоносителя видно, что во всем интервале температура гарантированно обеспечивает тепловой режим ЭРИ с тепловыделением не более 12 Вт, поскольку при низкой температуре теплоноситель перемерзает. Плоская ТT с ацетоном в качестве теплоносителя гарантированно обеспечивает тепловой режим ЭРИ с тепловыделением не более 10 Вт во всем диапазоне температур. Двухуровневая и трехуровневая СТТ с водой в качестве теплоносителя гарантированно обеспечивает тепловой режим ЭРИ с тепловыделением не более 40 Вт, что в 4 раза превышает характеристики одиночной ТТ. Трехуровневая СТТ с ацетоном в качестве теплоносителя обеспечивает тепловой режим ЭРИ с тепловыделением не более 28 Вт.

В результате проведенных исследований были установлены предельные значения отводимой тепловой мощности одиночной ТТ, двухуровневой и трехуровневой СТТ с разными теплоносителями. Подтверждена универсальность математической модели, представленной в работе [5], дополненной методом оптимизации.

\section{Список литературы / References}

[1] Amir Faghri. Frontiers in Heat Pipes (FHP), 2014, 5, 1. Global Digital Central, ISSN: $2155-$ 658X. DOI: 10.5098/fhp.5.1.

[2] Shukla K.N. Heat Pipe for Aerospace Applications - An Overview. Journal of Electronics Cooling and Thermal Control, 2015 5, 1-14. http://dx.doi.org/10.4236/jectc.2015.51001.

[3] Zohuri B. Application of Heat Pipe in Industry. Heat Pipe Design and Technology. Springer, Cham., 2016. https://doi.org/10.1007/978-3-319-29841-2.

[4] Дан П.Д., Рей Д.А. Тепловые трубы: пер. с англ. М.: Энергия, 1979. 272 с. [Dan P.D., Ray D.A. Heat Pipes. (transl. from English). M., Energiya, 1979. 272 p. (in Russian)]

[5] Деревянко В.А., Нестеров Д.А., Косенко В.Е. и др. Плоские тепловые трубы для отвода тепла от электронной аппаратуры в космических аппаратах. Вестник СибГАУ, 2013, 6(52), 111-116 [Derevyanko V.A., Nesterov D.A., Kosenko V.E., et al. Flat heat pipes for cooling spacecraft electronics, Vestnik SibGAU, 2013, 6(52), 111-116 (in Russian)]

[6] Лукс А.Л., Матвеев А.Г. Анализ основных расчетных и экспериментальных теплофизических характеристик аммиачных тепловых труб повышенной тепловой проводимости из алюминиевых сплавов. Вестник СамГУ. Естественнонаучная серия. 2008, 3(62), 331-357 [Luks A.L., Matveyev A.G. Comparative analysis of basic calculated and experimental thermal and physical characteristics of ammonia-Al alloy heat pipes with enhanced thermal conductivity, Vestnik SamGU. Natural science series, 2008, 3(62), 331-357 (in Russian)]

[7] Лукс А.Л., Матвеев А.Г., Зеленцов Д.В. Методика расчета тепловых труб, отводящих тепло от тепловыделяющей поверхности. Градостроительство и архитектура, 2018, 8, 1(30), 35-39 [Luks A.L., Matveyev A.G., Zelentsov D.V. Procedure to calculate heat pipes transporting heat from heat dissipating surface, Gradostroitelstvo i arkhitektura, 2018, 8, 1(30), 35-39 (in Russian)] 
[8] Козулин И.А. Экспериментальное исследование газожидкостного течения в микроканалах с различной ориентацией. Автореф. дис. ... канд. физ.-мат. наук. Новосибирск, 2013. 23 c. [Kozulin I.A. Experimental study of gas-liquid flow in microchannels with various orientations. Synopsis of thesis ... cand. phys.-math. Science. Novosibirsk, 2013. 23 p. (in Russian)]

[9] Кузнецов Г.В. Санду С.Ф. Численное моделирование теплофизических процессов в приборных отсеках современных искусственных спутниках Земли. Теплофизика и аэромеханика, 1998, 5(3) 469-477 [Kuznetsov G.V., Sandu S.F. Numerical modeling of thermal physical processes in instrument compartments of modern manmade Earth's satellites. Thermal Physics and Aeromechanics, 1998, 5(3), 469-477 (in Russian)]

[10] Kuznetsov G.V., Sandu S.F. Special features of the thermophysical modeling of instrument cubicles of spaceraft. Journal of Engineering Physics and Thermophysics, 2001, 74(6), 1431-1436.

[11] Китаев А.И., Лукс А.Л., Порядин А.В. Тепловые трубы повышенной тепловой проводимости - как базовые элементы системы терморегулирования в аэрокосмической технике. Вестник СамГУ, 2009, 3-2(19), 98-101 [Kitayev A.I., Luks A.L., Poryadin A.V. Heat pipees with enhanced thermal conductivity as basic elements of a thermal control system in aerospace equipment, Vestnik SamGU, 2009, 3-2(19), 98-101 (in Russian)]

[12] Бирюк В.В., Китаев А.И. Применение тепловых труб для охлаждения РЭА. Вестник СамГУ, 2009, 3-2(19), 342-345 [Biryuk V.V., Kitayev A.I. Heat pipe application in electronics cooling Применение тепловых труб для охлаждения РЭА, Vestnik Sam GU, 2009, 3-2(19), 342-345 (in Russian)]

[13] Кулагин В.А., Соколов Н.Ю. Разработка оборудования для тестирования тепловых труб. Журнал Сиб. федер. ун-та. Техника и технологии, 2015, 8(6), 774-785. DOI: 10.17516/1999494X-2015-8-6-774-785 [Kulagin V.A., Sokolov N.Yu. Development of equipment to test heat pipes J. Sib. Fed. Univ. Eng. technol. 2015, 8(6), 774-785. DOI: 10.17516/1999-494X-2015-8-6-774-785 (in Russian)].

[14] Кулагин В.А., Соколов Н.Ю. Совершенствование оборудования для проверки Т-образных плоских тепловых труб. Безопасность и живучесть технических систем: Материаль и доклады V Всероссийской конференщии: в 3 m. (Красноярск, 12-16 октября 2015 года). Красноярск: Сиб. федер. ун-т, 2015. Т. 2. 10-21 [Kulagin V.A., Sokolov N.Yu. Upgrading of testing equipment for T-shaped flat heat pipes. Safety and Survivability of Engineering Systems: Materials and Proceedings of V All-Russian conference: in 3 volumes. (Krasnoyarsk, October 12-16, 2015). Krasnoyarsk: Sib. Fed. Univ. 2015. V. 2. 10-21 (in Russian)]

[15] Kulagin V.A., Sokolov N.Yu. Improving the of thermophysical characteristics of heat pipes. J. Sib. Fed. Univ. Eng. technol. 2017, 10(3), 372-376. DOI: 10.17516/1999-494X-2017-10-3-372-376 [Kulagin V.A., Sokolov N.Yu. Improving thermophysical characteristics of heat pipes. J. Sib. Fed. Univ. Eng. technol. 2017, 10(3), 372-376. DOI: 10.17516/1999-494X-2017-10-3-372-376. (in Russian)].

[16] Кулагин В.А., Соколов Н.Ю. Численное исследование характеристик тепловых труб в составе радиоэлектронного оборудования космических аппаратов. Журнал Сиб. федер. унта. Техника и технологии, 2015, 8(6), 769-773. DOI: 10.17516/1999-494X-2015-8-6-769-773 [Kulagin V.A., Sokolov N.Yu. Numerical analysis of characteristics of heat pipes in spacecraft electronics. J. Sib. Fed. Univ. Eng. technol. 2015, 8(6), 769-773. DOI: 10.17516/1999-494X-2015-8-6-769-773 (in Russian)] 
[17] Кулагин В.А., Соколов Н.Ю. Математическое моделирование режимов работы тепловых труб в составе радиоэлектронного оборудования космических аппаратов. Безопасность и живучесть технических систем: Матер. и докл. V Всерос. конф.: в 3 m. (Красноярск, 12-16 октября 2015 года). Красноярск: Сиб. федер. ун-т, 2015. T. 2. 6-10 [Kulagin V.A., Sokolov N.Yu. Mathematical modeling of operating modes of heat pipes in the composition of radio-electronic equipment of spacecraft, Safety and survivability of technical systems: Mater. and reports. V All-Russian Conf.: in 3 vol. (Krasnoyarsk, October 12-16, 2015). Krasnoyarsk: Sib. Feder. un-t, 2015. T. 2. 6-10 (in Russian)]

[18] Кулагин В.А., Соколов Н.Ю. Физико-математическое моделирование предельных характеристик системы тепловых труб в составе радиоэлектронного оборудования космических аппаратов. Безопасность и мониторинг техногенных и природных систем. Матер. и докл. VI Всерос. конф. (Красноярск, 18-21 сент. 2018 г.), Красноярск: СФУ, 2018. 205-210 [Kulagin V.A., Sokolov N.Yu. Physical mathematical modeling of operating limits of a system of heat pipes in spacecraft electronics. Safety and monitoring of technogenic and natural systems: Materials and Proceedings of VI All-Russian Conf. (Krasnoyarsk, Sept.18-21, 2018), Krasnoyarsk: SFU, 2018. 205-210 (in Russian)]

[19] Пат. 2403692 РФ, МПК Н05К 1/00, Н05К 7/20. Модуль радиоэлектронной аппаратуры с гипертеплопроводящим основанием. Сунцов С.Б., Косенко В.Е., Деревянко В.А.; заявитель и патентообладатель Открытое акционерное общество «Информационные спутниковые системы» имени академика М.Ф. Решетнева». - № 2009116488/07; заявл. 29.04.2009; опубл. 10.11.2010, Бюл. № 31 [Patent 2403692 RF, МПК Н05К 1/00, H05К 7/20. Radio electronic module with a hyperheat-conducting base frame. Suntsov S.B., Kosenko V.E., Derevyanko V.A. Applicant and patent holder: M.F. Reshetnev «Information Satellite Systems» Open Joint-Stock Company. - No. 2009116488/07; Appl. 29.04.2009; Publ. 10.11.2010, Bul. № 31 (in Russian)]

[20] Чи С. Тепловые трубы: теория и практика. М.: Машиностроение, 1981. 207 с. [Chi S. Heat pipes: theory and practice. M., Mashinostroyeniye, 1981. 207 p. (in Russian)] 